\title{
SIMULAÇÃO DE MICRODESGASTE POR DESLIZAMENTO LINEAR RECÍPROCO: ESTUDOS INICIAIS ENVOLVENDO A ESFERA
}

P Y. Villamizar ${ }^{(1)}$ (yesenia.villamizar@ufrgs.br), D.Giarollo ${ }^{(1)}$ (danielagiarollo@hotmail.com), C. Mazzaferro ${ }^{(1)}$ (cintia.mazzaferro@ufrgs.br)

(1) Universidade Federal do Rio Grande do Sul (UFRGS); Programa de Pós-graduação em Engenharia Mecânica.

RESUMO: O método dos elementos finitos (MEF) pode ser utilizado como uma ferramenta para fazer previsão das características de desgaste dos materiais. Essa previsão do comportamento do material sob carga permite economizar recursos experimentais e reduzir a porcentagem do erro. Neste trabalho se apresenta uma abordagem numérica sobre o fenômeno de desgaste comparando seus resultados com testes experimentais. O modelo foi desenvolvido no software comercial COMSOL Multiphysics. O caso de estudo foi o deslizamento linear reciproco de uma esfera de alumina sobre uma placa de um aço de alta resistência e baixa liga. Se utilizou a lei de desgaste de Archard, onde a perda de altura nodal correspondente na esfera foi calculada segundo a taxa de desgaste do material, pressão aplicada e distância de deslizamento incremental. Os resultados das simulações apresentaram concordância com os fenômenos físicos envolvidos no processo de desgaste, mas a comparação dos valores observados na simulação com aqueles de testes experimentais mostrou um erro máximo de $24 \%$. Nas próximas etapas de estudo, o modelo numérico deverá ser aperfeiçoado e o comportamento em desgaste da esfera assim como da placa de aço será analisado.

PALAVRAS-CHAVE: SIMULAÇÃO DE DESGASTE, tAXA DE DESGASTE, PRESSÃO DE CONTATO, MEF, PROFUNDIDADE DESGASTADA.

\section{SIMULATION OF MICRO-WEAR BY RECIPROCAL LINEAR SLIDING: INITIAL STUDIES INVOLVING THE SPHERE}

ABSTRACT: The finite element method (FEM) is used as a tool for forecasting the wearing features of materials. This can contribute to save experimental resources and decrease the error rates. In this work is shown a numerical approach about the wear phenomena, comparing their results with experimental data. The model was developed in COMSOL Multiphysics simulating the linearly reciprocal ball-on-flat sliding wear. The plate material is a high strength low alloy steel and alumina was employed for the ball. The Archard law of wear was used. The nodal height losses for the ball were calculated through the specific wear rate, the pressure, and the incremental sliding distance. The simulation results demonstrated concordance with the physical phenomena involved in the wear process, but the comparison of simulation results and experimental data showed a maximum error of $24 \%$. The improvement of numerical modelling and analyses of the wear behavior of ball as well as steel plate are the next stage of this study.

KEYWORDS: WEAR SIMULATION, WEAR RATE, CONTACT PRESSURE, MEF, WEAR DEPTH 


\section{0 \\ XX CONEMI}

\section{INTRODUÇÃO}

O desgaste deslizante é um dos tipos mais comuns de desgaste que ocorre em diferentes contatos mecânicos. O deslizamento envolve componentes que têm superfícies em contato que por sua vez sofrem movimentos relativos entre as peças, os quais introduzem desgastes. Após um período de tempo esses desgastes podem alterar a cinemática do deslizamento podendo causar falhas nos componentes envolvidos.

A previsão numérica e a comparação com simulações são um bom complemento para obter mais informações sobre os fatores que afetam e controlam o desgaste. As primeiras pesquisas sobre modelos para determinar o desgaste usaram a equação de Archard. Archard, 1953 apresentou um método para estimar o volume de desgaste em qualquer situação tribológica particular, tanto para contatos adesivos quanto para abrasivos.

A metodologia mais comum para prever o fenômeno de desgaste é o modelo incremental. O modelo inclui um procedimento iterativo onde a distribuição de pressão entre as superfícies de contato é avaliada e usada para calcular a profundidade do desgaste em pontos discretos na superfície de acordo com a lei de desgaste de Archard. A geometria de cada superfície é atualizada, a partir da nova pressão de contato e é reaplicada. Mediante uma discretização se integra o avanço do desgaste ao longo do tempo.

Os primeiros modelos de desgaste incremental usando uma análise experimental em conjunto com elementos finitos foram feitos por Jonansson, 1994 que adicionou o conceito de distribuição de pressão no contexto de desgaste, anteriormente desenvolvida por Hertz, nos seus trabalhos da tensão de contato de dois sólidos elásticos. Hertz considerou os corpos em contato como meios-espaços elásticos cujo perfil de contato é elipsoidal, sem atrito e sem formação de tensões de Hertz (Johnson, 1982). Essa abordagem tem sido usada frequentemente em cálculos de tensão de contato. O desgaste ocorre quando as superfícies dos componentes mecânicos entram em contato umas com as outras, a questão de grande importância prática é a quantidade de material que será perdido durante o tempo de operação determinado (Johnson, 1985).

Outra pesquisa representativa foi feita por Põdra e Andersson, 1999 os quais sugerem que os parâmetros que influenciam a taxa de desgaste $(k)$ pelo deslizamento são a dureza dos materiais, e a carga aplicada.

Ao longo do tempo os pesquisadores melhoraram as análises e predições de desgaste onde propõem diversos métodos para maior eficácia dos modelos baseando-se na teoria de Archard. Hegadekatte et al., 2008 usaram métodos numéricos incorporando um modelo numérico de 
mecânica de contato conhecido como modelo de superfície de Winkler, que descreve essas instabilidades numéricas em termos dos componentes de dispersão da distribuição de pressão integrada. Mukras et al., 2009 sugerem variar iterativamente a magnitude da extrapolação, para otimizar a velocidade de integração, minimizando o erro numérico de modo que a suavidade geral da distribuição de pressão permaneça. Também recomendam o uso de computação paralela onde diferentes processadores resolvam modelos de elementos finitos idênticos ao mesmo tempo. Estudos como os desenvolvidos por Telliskivi, 2004 e Rudnytskyj, 2018 implementaram essa metodologia para simular os efeitos de desgaste de um elemento rodante e um bloco-sobre-plano respetivamente obtendo resultados satisfatórios.

Dessa forma o método de elementos finitos (MEF) é uma ferramenta versátil para resolver problemas de tensão e deformação, independentemente da geometria dos corpos. Nesse artigo foram empregados os softwares COMSOL Multiphysics 5.2a e LiveLink ${ }^{\mathrm{TM}}$ MATLAB $^{\circledR}$ para determinar a pressão de contato e simular o desgaste da esfera de alumina sobre uma placa de aço. As simulações numéricas foram comparadas com dados experimentais obtidos em ensaios de microdesgaste por deslizamento linear recíproco.

\subsection{Modelagem de desgaste}

A lei de Archard calcula o volume desgastado $V e[\mathrm{~m} 3]$ em função da distância total de deslizamento $s[m]$, carga normal $F n[N]$, dureza do material mais macio $H[P a]$ e coeficiente de desgaste $K$ como é mostrado na Equação (1). O coeficiente de desgaste não dimensionado $K$ e a dureza são agrupados em um coeficiente de desgaste unidimensional $k[\mathrm{~Pa}-1]$ (Stachowiak et al., 2014).

$$
\frac{V_{e}}{S}=K \frac{F n}{H}
$$

Um parâmetro importante para modelagem de desgaste é a profundidade de desgaste $h$, calculada dividindo o primeiro termo da Equação $1\left(\frac{V_{e}}{s}\right)$ pela área de contato (Equação (2)). $h$ é função da pressão de contato normal $p$ e o deslizamento total $s$ (Shen et al., 2010).

$$
h=k * p * s
$$




\section{CONEMI}

Onde $k\left[\frac{m^{2}}{N}\right]$ é a taxa especifica de desgaste (Totten et al., 2017). Baseado em observações experimentais o $k$ é descrito na Equação (3) (Stachowiak et al., 2014).

$$
k=\frac{V_{e}}{F n * S}
$$

A teoria de contato elástico de Hertz tem sido utilizada para cálculos de distribuição de tensões de contato. Porém, o modelo de contato de Hertz considera que todos os corpos são elásticos, o contato entre eles é elíptico, sem atrito. O módulo de Young reduzido equivalente $\left(E^{\prime}\right)$ é calculado mediante a Equação (4) (Zum Gahr, 1987)

$$
E^{\prime}=\left(\frac{1-\vartheta_{\text {esfera }}{ }^{2}}{E_{\text {esfera }}}+\frac{1-\vartheta_{\text {Placa }}{ }^{2}}{E_{\text {placa }}}\right)^{-1}
$$

Onde $E$ é o modulo de Young [Pa] e $\vartheta$ é coeficiente de Poisson dos materiais dos corpos em contato.

A pressão hertziana é distribuída hemisfericamente sobre a área de contato do plano, com um raio de contato $a$ no primero instante do contato como é mostrado na Figura 1 e calculada pela Equação (5), e é função do raio da esfera $R$ [m] (Ghosh, 2016).

$$
a=\left(\frac{3 F n * R}{4 E^{\prime}}\right)^{1 / 3}
$$

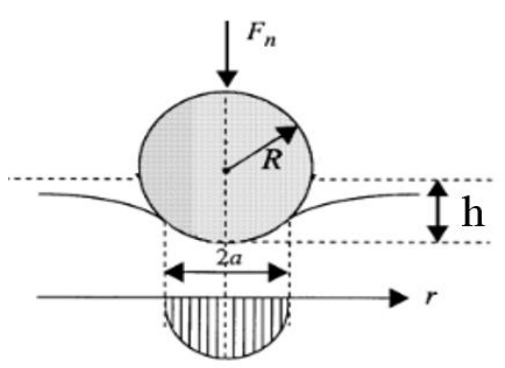

FIGURA 1. Esquema da distribuição de tensões para o contato elástico de esfera-plano. Fonte:

Autor (2020) 
A distribuição normal da pressão de contato Pmax depende da carga aplicada e do raio de contato conforme mostra a Equação (6) (Ghosh, 2016).

$$
P_{\max }=\frac{3 F n}{2 \pi a^{2}}
$$

O MEF pode ser usado como um método alternativo para determinar a pressão de contato, o qual considera os corpos como conjuntos de elementos discretos, e procura soluções para as equações diferenciais que satisfazem as condições de contorno.

\section{MATERIAIS E MÉTODOS}

Foi analisado o modelo numérico nos softwares COMSOL Multiphysics $5.2 a^{\circledR}$ e LiveLink $^{\mathrm{TM}}$ MATLAB ${ }^{\circledR}$. O modelo MEF usa uma aproximação de deformação planar, isto para avaliar a distribuição de pressão de contato com base em um modelo elástico linear (Kang, 2010; Rudnytskyj, 2018; Sutton, 2014). Como parte da modelagem numérica foi desenhado um cilindro 2D, para aproximar o fenômeno somente a um contato de tipo linha. Em 2D, o COMSOL só pode aproximar a uma linha de contato pois não é suportado um eixo de simetria axial (Kang, 2010; Sutton, 2014). A tensão residual dentro da placa não foi levada em consideração. As propriedades mecânicas dos materiais da esfera de alumina e da placa de aço são mostradas na Tabela 1.

TABELA 1. Propriedades mecânicas da esfera e placa utilizadas nas simulações

\begin{tabular}{|lcc|}
\hline & Esfera $\left(\mathrm{Al}_{2} \mathrm{O}_{3}\right)$ & Placa (Aço) \\
Módulo de Young & $300 e 9[\mathrm{~Pa}]$ & $200 e 9[\mathrm{~Pa}]$ \\
Coeficiente de Poisson & 0,21 & 0,30 \\
Densidade & $3961\left[\mathrm{~kg} / \mathrm{m}^{\wedge} 3\right]$ & $7850\left[\mathrm{~kg} / \mathrm{m}^{\wedge} 3\right]$ \\
\hline
\end{tabular}

Fonte: Autor (2020)

Na geometria do modelo, o comprimento do contato cilíndrico é de 0,054 mm que se considera como um ponto de contato entre a esfera e a placa, já que com esse valor é conservado o valor de esforço máximo do contato esfera e placa. Na Tabela 2 são apresentados os parâmetros da simulação. Foi definido o diâmetro de contato inicial de 0,103 mm de acordo com o cálculo do ponto 
de contato hertziano equivalente. Mediante a análise analítica de Hertz foi calculada a pressão máxima de contato o que permitiu validar o resultado de pressão de contato máximo teórico.

Se aplicaram condições de esfera rígida e placa deformável aos corpos de estudo. Se aplicou uma carga normal no limite superior da esfera de alumina, enquanto a parte inferior (placa de aço) é fixada em todas as direções (Figura 3). As cargas se estabeleceram segundo os testes experimentais de microdesgaste por deslizamento linear recíproco conduzidos por Giarollo, 2018, utilizando um microtribômetro pertencente ao Laboratório de Soldagem e Técnicas Conexas (LS\&TC) da Universidade Federal Rio Grande do Sul (UFRGS). A Figura 2 mostra o esquema do tribômetro e direção de deslizamento usado no teste experimental.
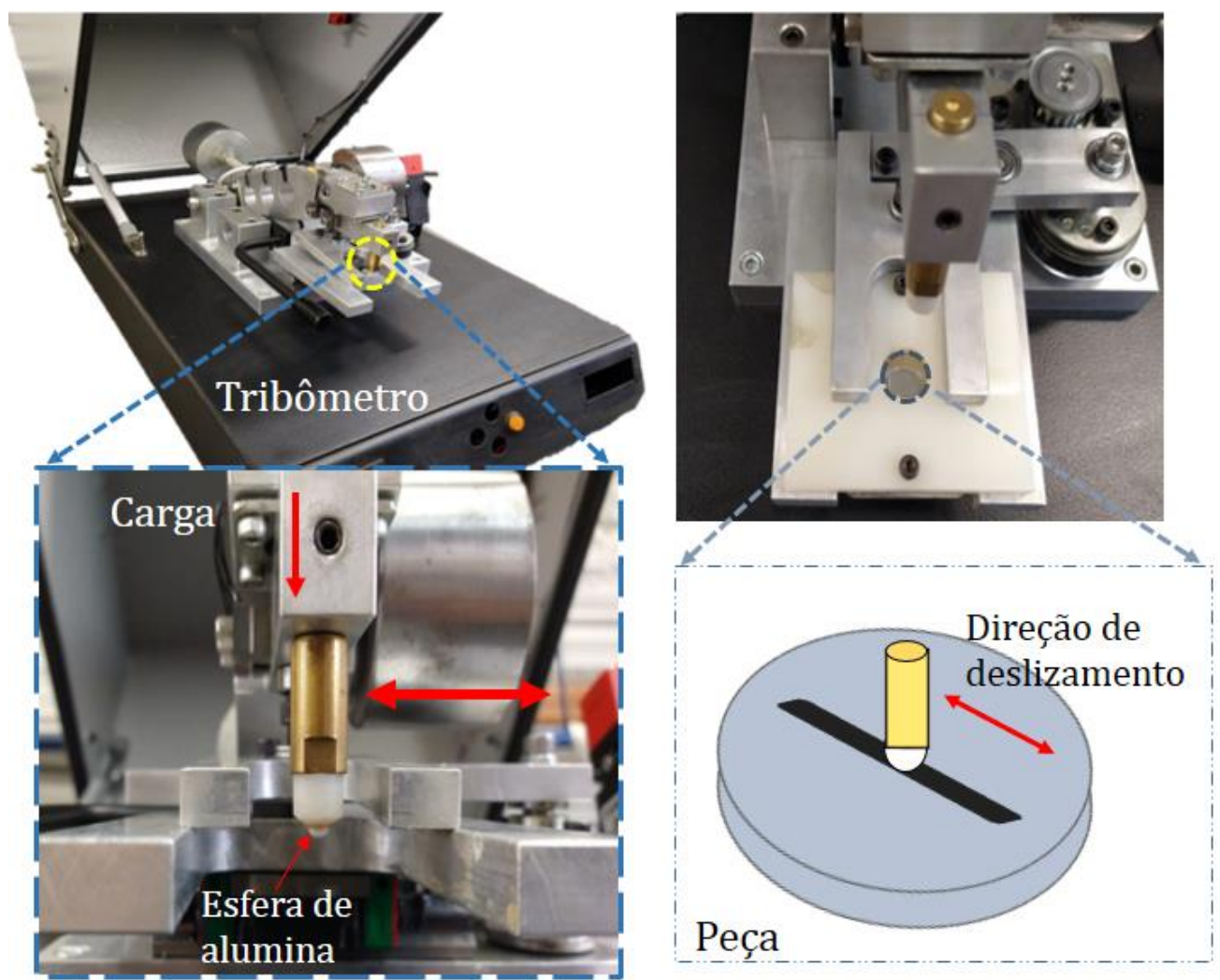

\section{Deslizamento recíproco}

FIGURA 2. Tribômetro utilizado nos testes experimentais de microdesgaste por deslizamento linear recíproco, mostrando alguns componentes e características de deslizamento. Fonte: Autor (2020).

Se definiu uma condição de contorno de tipo par de contato entre as geometrias do estudo (Figura 3), aumentando assim a eficiência computacional, já que o software predefine o cenário onde 
toda superfície entrará em contato com seu par, limitando a operação de análise de contato. (COMSOL, 2018). Essa mesma abordagem foi utilizada em trabalhos desenvolvidos por (Andersson; Almqvist; Larsson, 2011; Ghosh, 2016; Sutton, 2014).

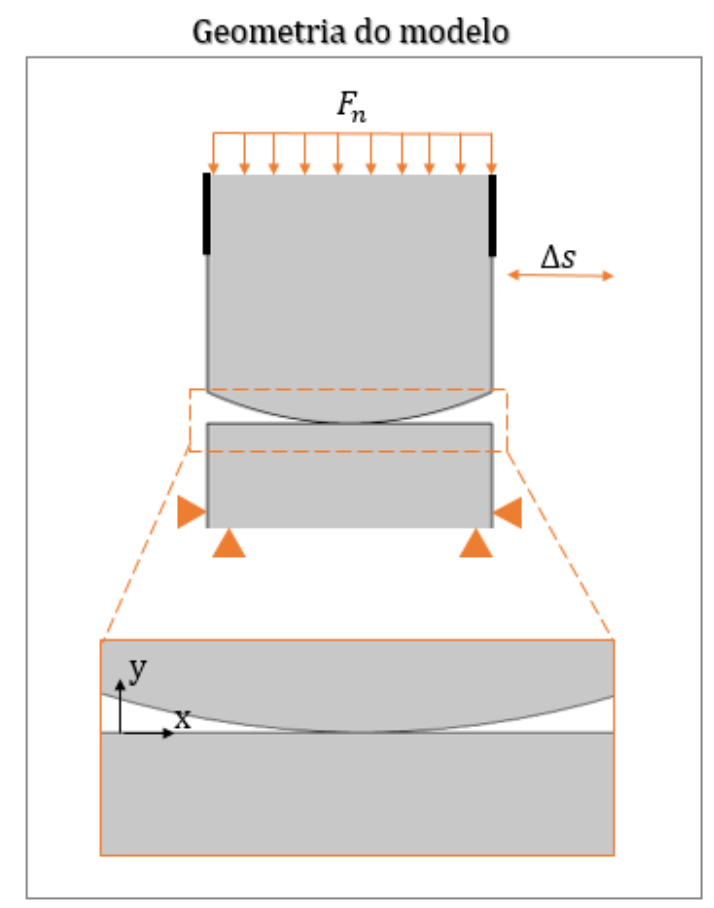

FIGURA 3. Esquema da geometria e das condições de contorno empregadas na simulação. Fonte: Autor (2020)

TABELA 2. Parâmetros utilizados na simulação, baseados em testes de desgaste linear-recíproco experimentais

\begin{tabular}{|cc|}
\hline \multicolumn{2}{|c|}{ Valores numéricos de experimentos na simulação } \\
\hline Força normal & $10 \mathrm{~N}$ \\
Amplitude da trilha & $2 \mathrm{~mm}$ \\
Taxa de desgaste & $1,721 \times 10^{-16} \mathrm{~m}^{2} / \mathrm{N}$ \\
Diâmetro da esfera & $4,762 \mathrm{~mm}$ \\
Velocidade linear & $4 \mathrm{~mm} / \mathrm{s}$ \\
\hline
\end{tabular}

Fonte: Autor (2020)

Foi empregado o método Augmented Lagrangian o qual garante que não haverá penetração entre os objetos em contato em uma solução bem convergente. Além disso, a pressão de contato e 


\section{0 \\ XX CONEMI}

FENEMI

as forças de friç̧ão são adicionadas como graus de liberdade (COMSOL, 2018). Outro aspecto importante refere-se ao problema inicial irrestrito; o recurso Spring Foundation é aplicado na parte superior da esfera para atribuir condições de limite elástico e de amortecimento ao estudo (COMSOL, 2020).

Na Figura 4 mostra-se o esquema da malha e sua condição mais fina na interface de contato. Quanto maior o número de elementos próximos ao contato, os resultados serão mais próximos da realidade dentro dos limites apropriados. A máxima profundidade onde ocorre a pressão máxima foi calculada segundo a formulação de Hertz para criar a região de maior refinamento da malha, no caso do cilindro foi de 0,016 $\mathrm{mm}$ e da placa 0,052 $\mathrm{mm}$. O tipo de elemento empregado na malha é triangular, o tamanho mínimo de elemento foi de 4×10-6 m e o número total de elementos foi 2463. Esse tamanho de elemento na zona de contato forneceu uma boa precisão dos resultados da pressão de contato normal, levando em consideração um tempo computacional aceitável.

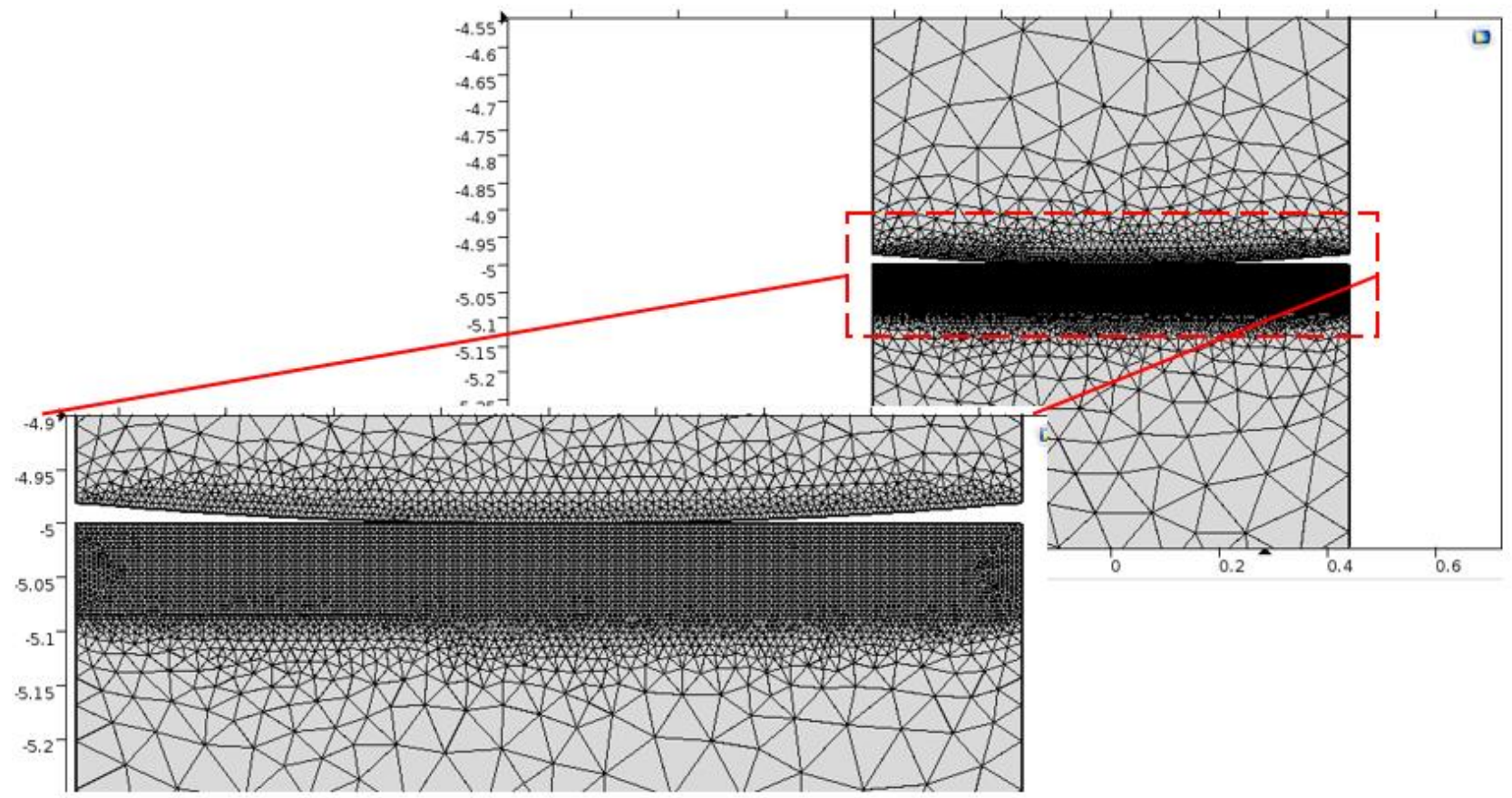

FIGURA 4. Malha empregada, com dimensões relacionadas ao refinamento no contato baseadas nos valores de profundidade de tensão máxima obtidos considerando a mecânica de contato Hertz. Fonte: Autor (2020).

Os incrementos de desgaste foram calculados por meio da Equação (2). O coeficiente de desgaste foi constante, igual a $k=1,721 \times 10-16 \mathrm{~m} 2 / N$ como mostra a Tabela 2. Esse valor foi calculado baseado no trabalho experimental desenvolvido por Giarollo, 2018. 
O algoritmo de trabalho do modelo de desgaste da esfera é apresentado na Figura 5. Inicialmente a geometria da esfera é criada por meio um arquivo de texto que contém as coordenadas dos pontos de controle, esse arquivo é usado no COMSOL Multiphycs para construir as condições de contato. A informação da pressão de contato é executada a partir do COMSOL, e com o auxílio do MATLAB o modelo de desgaste é executado conhecendo a pressão de contato. A atualização da geometria é realizada de acordo com $\Delta h$ desejado programado no código MATLAB.

Em seguida, o próximo ciclo de simulação é preparado criando um novo arquivo de texto com as coordenadas atualizadas dos pontos de controle que serão utilizados na configuração do modelo. O critério de parada refere-se a um número de ciclos que se deseja simular, então o procedimento funciona como um loop no MATLAB dentro do qual as simulações são calculadas no COMSOL. Todo o procedimento é controlado na interface LiveLink MATLAB do COMSOL.

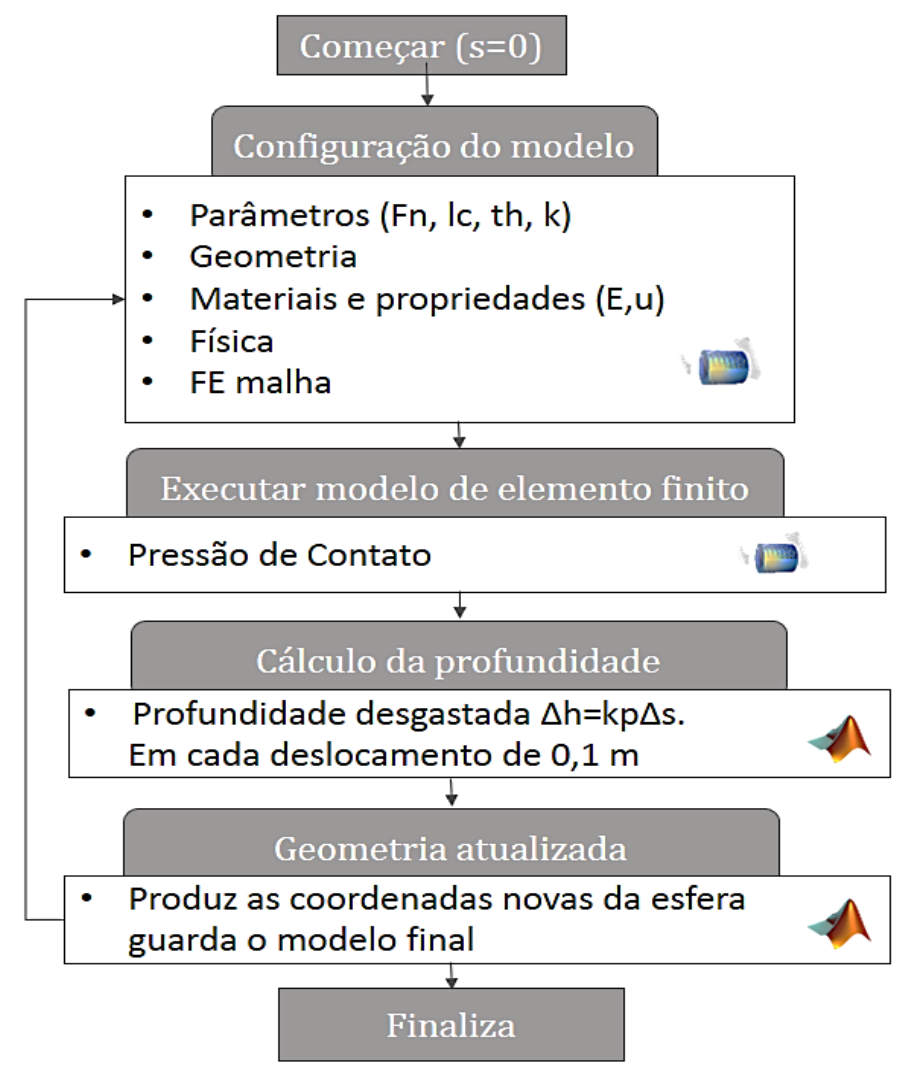

FIGURA 5. Algoritmo de trabalho empregado neste estudo. Fonte: Autor (2020).

Para a medição do volume desgastado nos testes experimentais na esfera, segundo Giarollo, 2018, foram feitas fotos das marcas deixadas na esfera com um microscópio óptico (Figura 6a). 
Posteriormente estas imagens foram analisadas em um programa edição para medição da marca como mostra a Figura 6b, onde obtiveram o diâmetro efetivo (D). Na Figura 6c mostra o volume desgastado $(V e)$, para o diâmetro efetivo da marca $\mathrm{D}$ foi encontrado a partir da relação da altura do material removido ou profundidade desgastada (h) (Figura 6d), segundo a norma ASTM G-133.
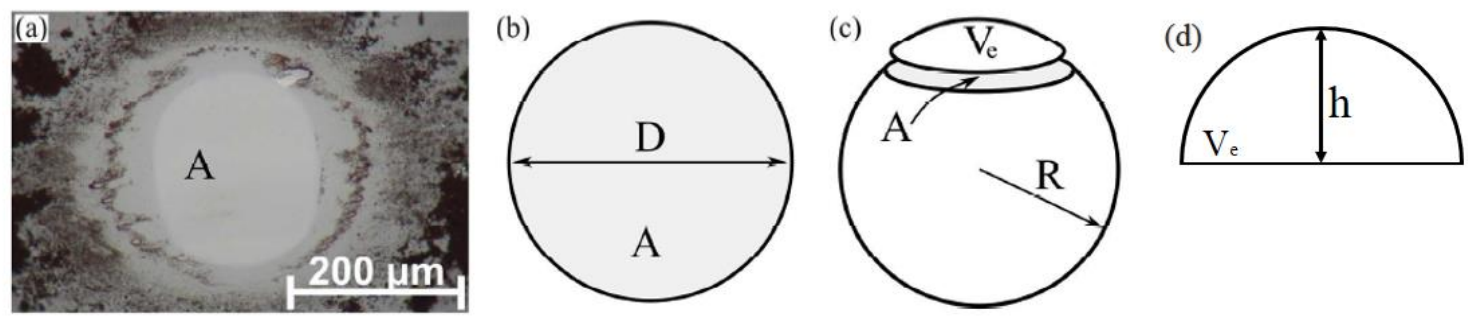

FIGURA 6 a) Imagens da esfera desgastada em ensaio experimental; b) Aproximação da área desgastada na esfera por um círculo de mesma área; c) Cálculo do volume desgastado na esfera; d)

Representação de profundidade h do volume desgastado Ve. Fonte: Adaptado (Giarollo, 2018).

\section{RESULTADOS}

Na Figura 7 estão apresentados os resultados da simulação da profundidade desgastada da esfera ao longo do tempo. A análise do perfil simulado de desgaste mostra semelhanças em relação aos experimentos. No instante zero, simbolizado pela linha azul, representa a esfera sem desgaste. À medida que o deslizamento avança, o desgaste ocorre no centro da face da esfera como mostra a linha amarela, até ficar plano, assim mesmo o raio da marca de desgaste, ou seja, os diâmetros efetivos representados pelas linhas vermelha e verde aumentam conforme avança o tempo. O valor final da profundidade de desgaste na esfera e o diâmetro efetivo do teste experimental com os dados da simulação foram comparados (Tabela 3). A profundidade de desgaste foi similar ao valor do teste experimental com uma diferença ao redor de $24 \%$, enquanto ao diâmetro efetivo a diferença foi de $18 \%$. 


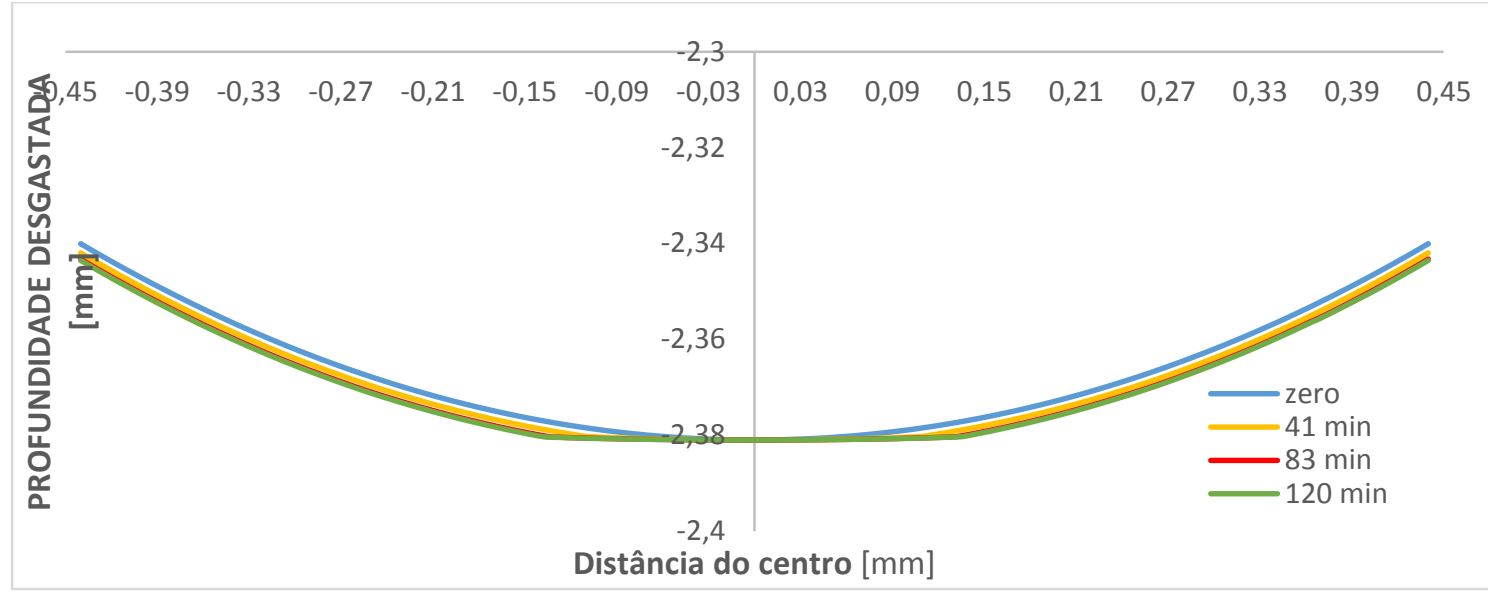

FIGURA 7. Resultados de simulação da profundidade de desgaste da esfera ao longo do tempo. Fonte: Autor (2020)

TABELA 3. Comparação da simulação e resultados experimentais para profundidade e diâmetro de desgaste da esfera, após a duração total de teste (2 h=120 min).

\begin{tabular}{l|l|ll}
\multicolumn{4}{c}{ Simulação desgaste MB } \\
\hline \multicolumn{2}{l}{ Profundidade desgastada (h) } & & \\
\hline simulação & $0,00341[\mathrm{~mm}]$ & Erro & \\
\hline Teste experimental & $0,00257[\mathrm{~mm}]$ & & $24 \%$ \\
\hline Diâmetro efetivo (D) & & & \\
\hline simulação & $0,27280[\mathrm{~mm}]$ & Erro & \\
\hline Teste experimental & $0,22139[\mathrm{~mm}]$ & & $18 \%$ \\
Fonte: Autor (2020) & & & \\
\end{tabular}

A pressão de contato durante o deslizamento não pode ser controlada facilmente em um experimento. A informação em tempo real da distribuição de pressão é um valioso dado do modelo numérico, que complementa as informações obtidas nos experimentos. A evolução da distribuição de pressão de contato e tensões de Von Mises em função do tempo obtidas por simulação são mostradas nas Figuras 8, 9, 10 e 11.

A Figura 8b mostra no primeiro instante a pressão de contato, que tem um comportamento parabólico, cujo valor máximo é de $1798 \mathrm{MPa}$, muito próximo ao comparado com a pressão teórica (analítica) calculada de 1787,1 MPa. Na Figura 8a expõe o comportamento da tensão de Von Mises na esfera e a placa, com maior concentração no centro da esfera, além disso ressalta a pressão de contato em forma de linha concentrada no centro da face em contato. 


\section{CONEMI}

FENEMI

O perfil de pressão de contato tende a reduzir o pico e aumentar a área de contato como se observa na Figura 9b à 41 min após começar o teste. O pico de pressão de contato nesse caso foi de 970 MPa. Para o mesmo instante do tempo a tensão de Von Mises diminui no centro, mas começa a se concentrar nas bordas, devido que as tensões se concentram nas bordas, já que inicia o comportamento plano na esfera.

Transcorrido 83min do teste, se percebem irregularidades em ambos lados do pico da pressão de contato, ocasionadas pela área plana da esfera nesse instante (Figura 10b).

A Figura 11b representa o perfil de pressão de contato reduzido aos 120 min do início do teste, respetivamente a Figura 11a mostra o comportamento das tensões de Von Mises.

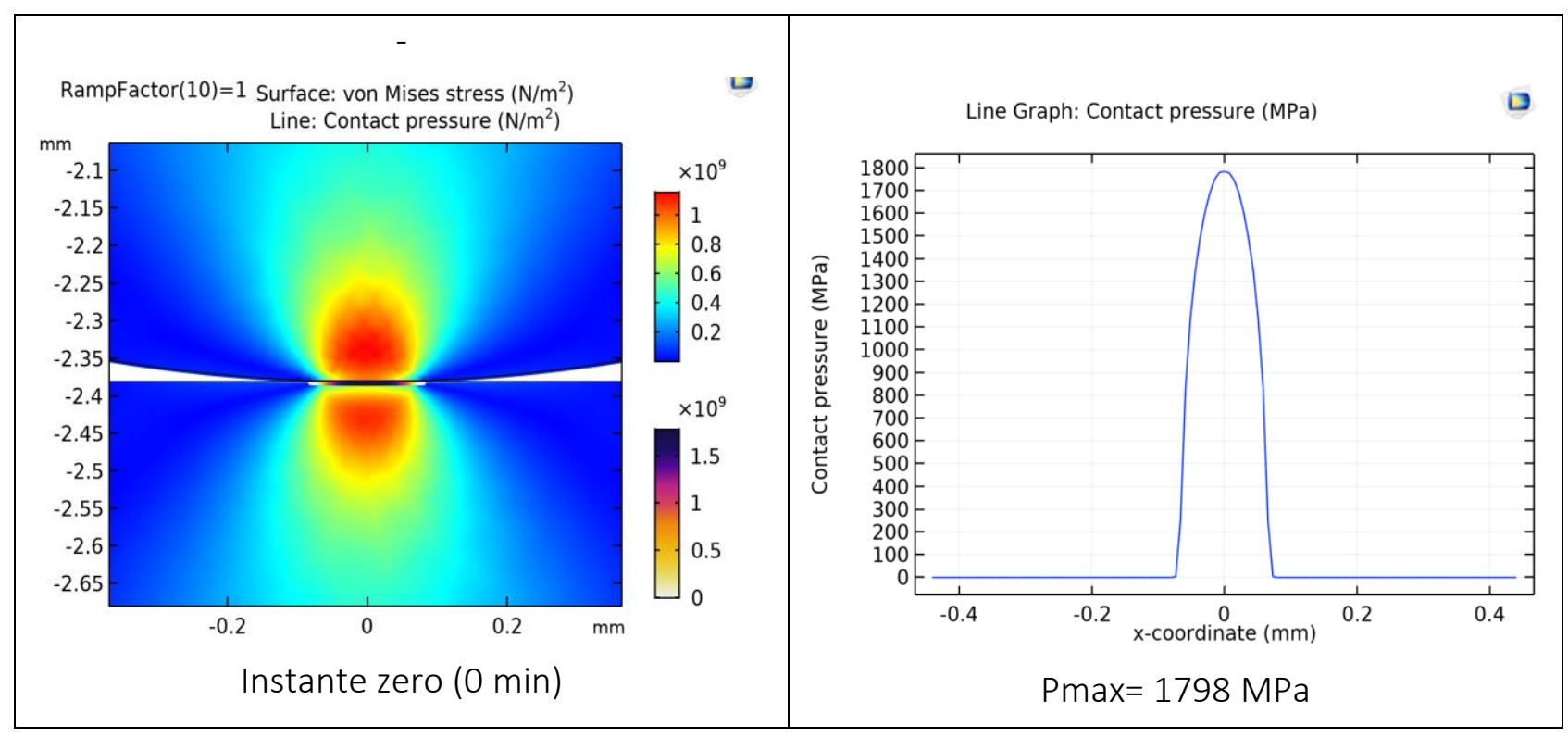

FIGURA 8. Resultados de simulação para tensão de Von Mises e pressão de contato no instante zero. Fonte: Autor (2020) 


\section{CONEMI}

FENEMI

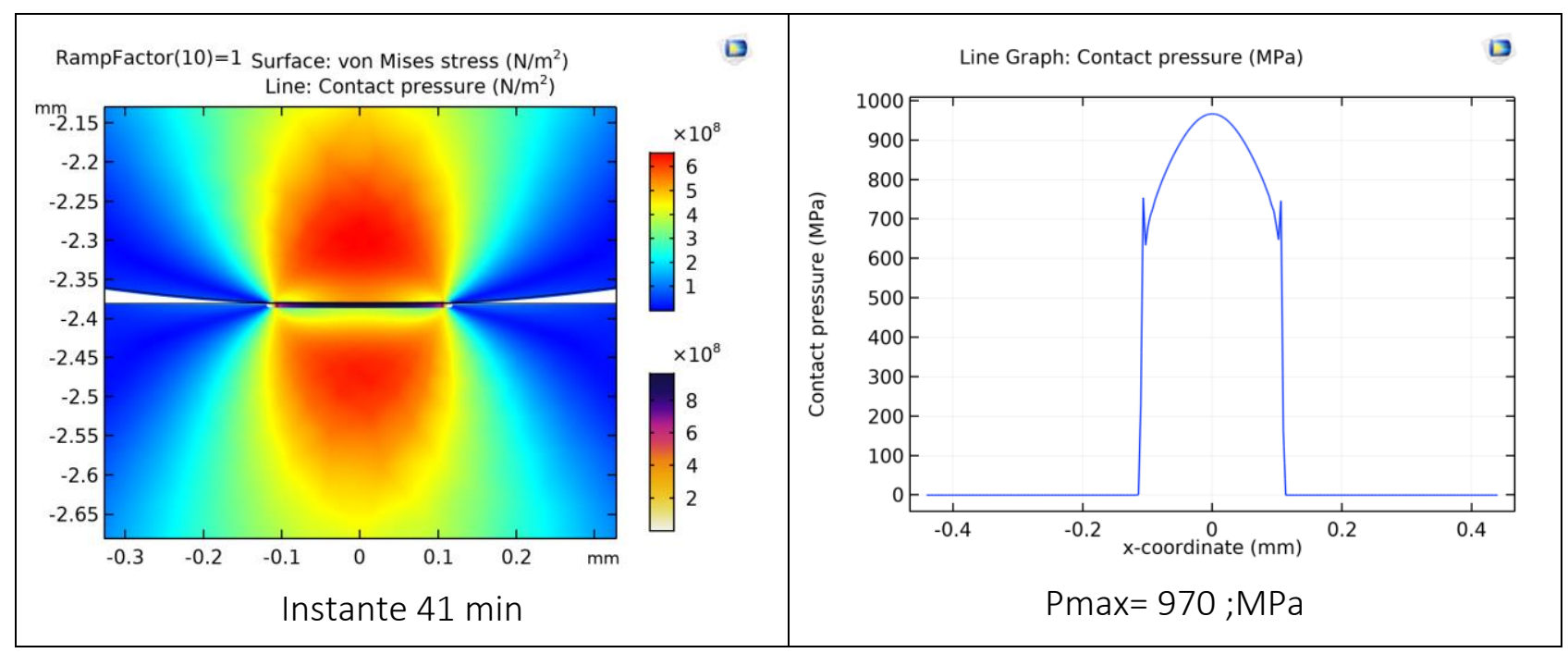

FIGURA 9. Resultados de simulação para tensão de Von Mises e pressão de contato aos 41 minutos do teste. Fonte: Autor (2020).

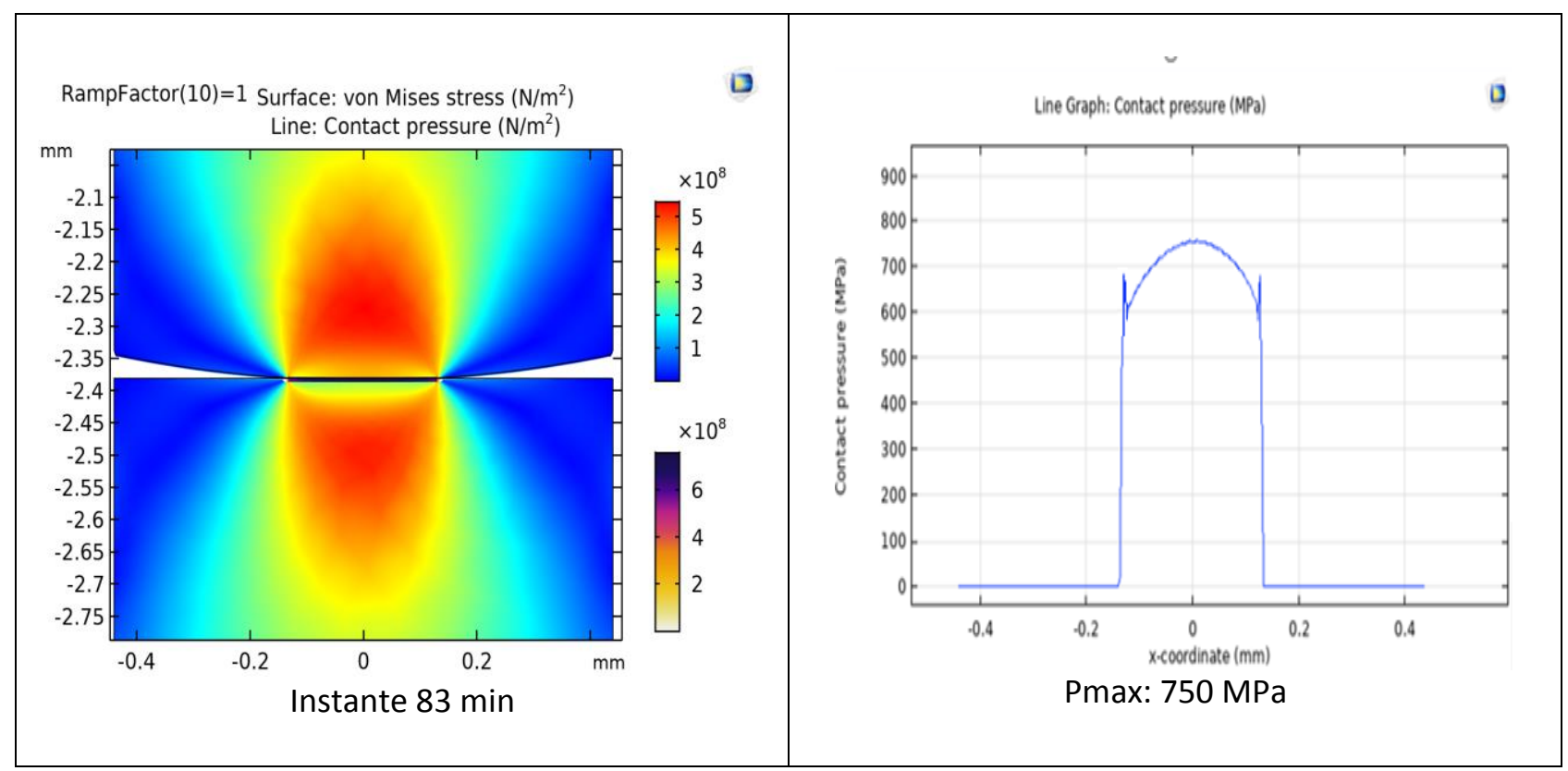

FIGURA 10. Resultados de simulação para tensão de Von Mises e pressão de contato aos 83 minutos do teste. Fonte: Autor (2020). 


\section{CONEMI}

FENEMI

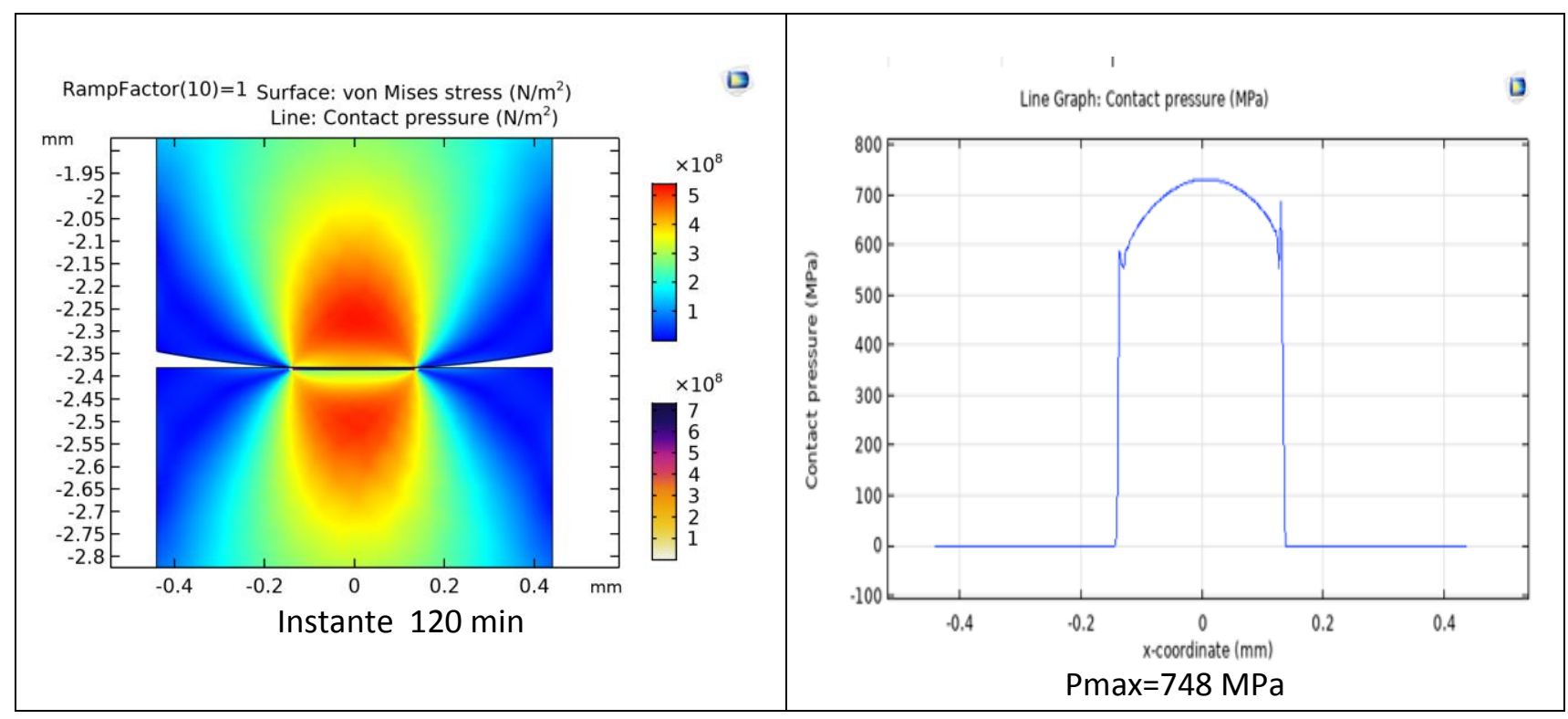

FIGURA 11 Resultados de simulação para tensão de Von Mises e pressão de contato aos 120 minutos do teste. Fonte: Autor (2020).

Pode-se observar que nas Figuras anteriormente referidas, a pressão de contato na região central do contato diminui ao longo do tempo enquanto a região de contato com pressões diferentes de zero se expande, dando um aumento do raio de contato, o que mostra que os resultados das simulações são condizentes fisicamente com os resultados experimentais. Porém, como já exposto, a diferença entre resultados de desgaste experimentais e simulados chega a 24\%, sendo necessário o aperfeiçoamento do modelo numérico para diminuição dos erros. Além disso, como próxima etapa para o estudo pretende-se também levar em conta o comportamento em desgaste da placa de aço.

\section{CONCLUSÕES}

O método proposto pode ser uma ferramenta aceitável para prever problemas de desgaste, empregando o modelo discretização quase estático, já que apresentou resultados condizentes com situações experimentais para os materiais aplicados no presente estudo, mas com diferenças de $24 \%$ na profundidade de desgaste e 18\% para o diâmetro efetivo (de desgaste) da esfera, que são valores 


\section{0 \\ XX CONEMI}

FENEMI

aceitáveis nesse estudo inicial mas que podem ser melhorados com aprimoramentos no modelo numérico empregado.

Os resultados de simulação mostraram que a profundidade de desgaste da esfera aumenta conforme aumenta a distância de deslizamento. Durante as primeiras etapas, a profundidade de desgaste aumenta rapidamente, devido ao valor da pressão de contato ser elevado, por ser menor a área de contato entre esfera e chapa. À medida que o tempo decorre, a velocidade com que aumenta a profundidade diminui, pois, a pressão de contato decresce simultaneamente com o aumento da área de contato.

\section{REFERÊNCIAS}

ANDERSSON, J.; ALMQVIST, A.; LARSSON, R. Numerical simulation of a wear experiment. Wear, v. 271, n. 11-12, pp. 2947-2952, 2011.

ARCHARD, J. F. Contact and rubbing of flat surfaces. Journal of Applied Physics, v. 24, n. 8, p. 981988, 1953.

COMSOL. RampFactor. 2020.

Disponível em: <https://br.comsol.com/support/knowledgebase/1102>.

COMSOL MULTIPHYSICS. Structural Mechanics Module. USA. Disponível em: www.comsol.de

GHOSH, A. Analytical investigation of fretting wear with special emphasis on stress based models. Tese Doutorado. Universidad Purdue, 82 p., 2016.

GIAROLLO, D. Influência do metal de adição na resistência ao desgaste abrasivo de dois aços estruturais. Dissertação de Mestrado em Engenharia Mecânica - Universidade Federal Rio Grande Do Sul UFRGS, 39-42p, 2018.

HEGADEKATTE, V. et al. A predictive modeling scheme for wear in tribometers. Tribology International, v. 41, n. 11, pp. 1020-1031, 2008.

JOHNSON, K. One hundred years of hertz contact. Cambridge University, v. 196, 363-378 p, 1982.

JOHNSON, K. L. Contact Mechanics. Cambridge ed. London New York.

KANG, S. Thesis - Friction and Elasto-Plastic deformation in Asperity Collision. Dissertação de Mestrado em Engenharia Mecânica - Universidad Tecnológica de Luleå, 54 p, 2010.

MUKRAS, S. et al. Numerical integration schemes and parallel computation for wear prediction using finite element method. Wear, v.266, pp. 822-831, 2009.

PÕDRA, P.; ANDERSSON, S. Simulating sliding wear with finite element method. Tribology International, v. 32, n. 2, pp. 71-81, 1999. 


\section{CONEMI}

FENEMI

RUDNYTSKYJ, A. Simulations of contact mechanics and wear of linearly reciprocating block-on-flat sliding test. Dissertação de Mestrado em Engenharia, Universidad Tecnológica de Luleå, 53 p, 2018.

STACHOWIAK, G.; BATCHELOR, A. Engineering Tribology. 4. Ed, ELSEVIER, 527p 2014.

SUTTON, D. Wear modelling of diamond-like carbon coatings against steel in deionised water. Tese de Doutorado. University of Southampton Faculty, 60 p, 2014.

TELLISKIVI, T. Simulation of wear in a rolling-sliding contact by a semi-Winkler model and the Archard's wear law. Wear, v. 256, n. 7-8, pp. 817-831, 2004.

TOTTEN, G. E. et al. ASM Handbook W Volume 18 Friction, Lubrication, and Wear Technology, Volume Editor Division Editors ASM International Staff Editorial Assistance. In: TOTTEN, G. E. (Ed.). ASM Handbook. International ed. Ohio. v. 18. Pp. 21.

ZUM GAHR, K. H. Microstructure and wear of materials. Tribology Series, Amsterdam, v. 10, 1987.

\section{AGRADECIMENTO}

A autora Yesenia Villamizar Gonzalez agradece ao Conselho Nacional de Desenvolvimento Científico e Tecnológico (CNPQ) pela bolsa de mestrado, e a autora Daniela Giarollo agradece à Coordenação de Aperfeiçoamento de Pessoal de Nível Superior (CAPES). 